\section{E-mental health}

\section{EPP0570}

\section{Insideout project: Using big data and machine learning for prevention in psychiatry}

F. Fiori Nastro ${ }^{1 \star}$, D. Croce $^{2}$, S. Schmidt ${ }^{3}$, R. Basili ${ }^{4}$ and

F. Schultze-Lutter ${ }^{5}$

${ }^{1}$ Department Of Systems Medicine, University of Rome "Tor Vergata”, Roma, Italy; ${ }^{2}$ Department Of Enterprise Engineering, University of Rome "Tor Vergata”, Roma, Italy; ${ }^{3}$ Clinical Psychology And Psychotherapy, University of Bern, Bern, Switzerland; ${ }^{4}$ Department Of Enterprise Engineering, University of Rome "Tor Vergata", Rome, Italy and ${ }^{5}$ Department Of Psychiatry And Psychotherapy, Heinrich-HeineUniversity, Düsseldorf, Germany

${ }^{*}$ Corresponding author.

doi: 10.1192/j.eurpsy.2021.919

Introduction: Social Media might represent an amazing and valuable source of information on mental health and well-being. Several researches revealed that adolescents aged 13 to 17 years old go "online" daily or stay online "almost constantly".

Objectives: The aim of this project is to identify distress in preclinical stages using Social media screening methods. The system can be modelled to centre on different several health-related topics. Methods: We created a digital system able to analyse scripts written by adolescents on Twitter. InsideOut works using machine learning techniques and computational linguistic items to catch significant and sense of written messages and it improves its performances with iterations. The system is able to automatically identify semantic information relevant to different topics: in this case "distress in teenagers".

Results: The task of our system is considered correct when it is able to identify triples of Life Event, Sentiment and Experience of a tweet in agreement with the Gold Standard established among the annotators. The system has around $70 \%$ of accuracy in identifying triples. The analysis has been carried out both in Italian and English collecting over 4 million Italian tweets and 30 million English tweets. Comparative analysis with self-report questionnaires show that tweet analysis is able to suggest similar statistics.

Conclusions: This study analyzed contents of messages posted on Social Media Twitter meta-dating them with psychological and health-related information. Using InsideOut, we can plan clinical intervention in district and regions where high levels of uneasiness are revealed.

Keywords: distress; prevention; machine learning; e-mental health

\section{EPP0569}

Pending challenges to e-mental health in the COVID-19 era: Acceptability of a smartphone-based ecological momentary assessment application among patients with schizophrenia spectrum disorders

J.-D. Lopez-Morinigo ${ }^{1 \star}$, B.-E. Maria Luisa ${ }^{2}$, A. Porras-Segovia ${ }^{3}$, A. Sánchez-Escribano Martínez ${ }^{3}$, P.-J. Escobedo-Aedo ${ }^{3}$, V. González Ruiz-Ruano ${ }^{3}$, L. Mata-Iturralde ${ }^{3}$, L. Muñoz-Lorenzo ${ }^{3}$, S. Sánchez-Alonso ${ }^{3}$, A. Artés-Rodríguez ${ }^{4}$ and E. Baca-Garcia
${ }^{1}$ Psychiatry, Universidad Autónoma de Madrid, Madrid, Spain;

${ }^{2}$ Psychiatry, Universidad Autonoma, Madrid, Spain; ${ }^{3}$ Psychiatry, Hospital Universitario Fundación Jiménez Díaz, Madrid, Spain and ${ }^{4}$ Signal Theory And Communications, Universidad Carlos III, Madrid, Spain

${ }^{\star}$ Corresponding author.

doi: 10.1192/j.eurpsy.2021.920

Introduction: Concerns have been raised about ecological momentary assessment (EMA) acceptability among patients with schizophrenia spectrum disorders (SSD), which is of major relevance during the e-Mental health-focused COVID-19 pandemic.

Objectives: To investigate i) the levels of adherence to a passive smartphone-based EMA tool, the Evidence-Based Behavior (eB2), among SSD patients; and ii) putative predictors of this.

Methods: Sample: SSD (F20-29-ICD10) outpatients, age 18-64, without financial incentives, recruited over 17/06/201911/03/2020 at the Hospital Universitario Fundación Jiménez Díaz (Madrid, Spain). Those who accepted the eB2 installation -usersand those who did not -non-users- were compared in sociodemographic, clinical, premorbid adjustment, neurocognitive, psychopathological, insight and metacognitive variables by a multivariable binary logistic regression model.

Results: Sample ( $\mathrm{N}=77)$ : $\mathrm{n}=41$ males; age: $47.69 \pm 9.76$ years, $\mathrm{n}=24$ users (31.2\%). $n=14$ users (70\%) had the eB2 installed at follow-up (median=14.50 weeks).

\begin{tabular}{|c|c|c|c|c|c|c|}
\hline \multicolumn{7}{|c|}{ Multivariable binary logistic regression model on 'user' as outcome } \\
\hline & $\beta$ & SE & Wald & $\mathrm{p}$ & OR & $95 \% \mathrm{Cl}$ \\
\hline Age & -0.075 & 0.038 & 3.910 & 0.048 & 0.928 & $\begin{array}{r}0.861- \\
0.999\end{array}$ \\
\hline Education level & -0.967 & 1.289 & 0.563 & 0.453 & 0.380 & $\begin{array}{r}0.030- \\
4.755\end{array}$ \\
\hline $\begin{array}{l}\text { Early adole } \\
\text { scence } \\
\text { premorbid } \\
\text { adjustment }\end{array}$ & -0.285 & 0.110 & 6.695 & 0.010 & 0.752 & $\begin{array}{r}0.606- \\
0.933\end{array}$ \\
\hline $\begin{array}{c}\text { Trail Making } \\
\text { Test A }\end{array}$ & -0.030 & 0.025 & 1.488 & 0.222 & 0.970 & $\begin{array}{r}0.924- \\
1.018\end{array}$ \\
\hline $\begin{array}{l}\text { Trail Making } \\
\text { Test B }\end{array}$ & -0.005 & 0.010 & 0.278 & 0.598 & 0.995 & $\begin{array}{r}0.976- \\
1.014\end{array}$ \\
\hline Cognitive Insight & 0.062 & 0.061 & 1.043 & 0.307 & 1.064 & $\begin{array}{l}0.944- \\
1.200\end{array}$ \\
\hline
\end{tabular}

$\mathrm{X}^{2}=25.296, \mathrm{df}=6, \mathrm{p}<0.001$. Nagelkerke- $\mathrm{R}^{2}=44.7 \%$. Correctly classified: $76.9 \%$, users: $54.5 \%$, non-users: $88.4 \%$.

Conclusions: Acceptability of a smartphone-based EMA application among SSD patients was low. Age (young) and good premorbid adjustment predicted acceptability. e-Mental Health methods need to be tailored for patients with SSD. Otherwise, these highly vulnerable individuals may be neglected by e-health-based services in the post-COVID-19 years ahead.

Keywords: Schizophrenia spectrum disorders; acceptability; ecological momentary assessment 